\title{
Hankel and Toeplitz transforms on $H$ 1: continuity, compactness and Fredholm properties
}

Article

Accepted Version

Papadimitrakis, M. and Virtanen, J. A. (2008) Hankel and Toeplitz transforms on $\mathrm{H} 1$ : continuity, compactness and Fredholm properties. Integral Equations and Operator Theory, 61 (4). pp. 573-591. ISSN 0378-620X doi:

https://doi.org/10.1007/s00020-008-1609-2 Available at https://centaur.reading.ac.uk/29128/

It is advisable to refer to the publisher's version if you intend to cite from the work. See Guidance on citing.

Published version at: http://dx.doi.org/10.1007/s00020-008-1609-2

To link to this article DOI: http://dx.doi.org/10.1007/s00020-008-1609-2

Publisher: Springer

Publisher statement: The original publication is available at www.springerlink.com (see DOI link above).

All outputs in CentAUR are protected by Intellectual Property Rights law, including copyright law. Copyright and IPR is retained by the creators or other copyright holders. Terms and conditions for use of this material are defined in the End User Agreement.

www.reading.ac.uk/centaur 


\section{CentAUR}

Central Archive at the University of Reading

Reading's research outputs online 


\title{
HANKEL AND TOEPLITZ TRANSFORMS ON $H^{1}$ : CONTINUITY, COMPACTNESS AND FREDHOLM PROPERTIES
}

\author{
M. PAPADIMITRAKIS AND J. A. VIRTANEN
}

\begin{abstract}
We revisit the boundedness of Hankel and Toeplitz operators acting on the Hardy space $H^{1}$ and give a new proof of the old result stating that the Hankel operator $H_{a}$ is bounded if and only if $a$ has bounded logarithmic mean oscillation. We also establish a sufficient and necessary condition for $H_{a}$ to be compact on $H^{1}$. The Fredholm properties of Toeplitz operators on $H^{1}$ are studied for symbols in a Banach algebra similar to $C+H^{\infty}$ under mild additional conditions caused by the differences in the boundedness of Toeplitz operators acting on $H^{1}$ and $H^{2}$.
\end{abstract}

\section{Introduction AND MAIN RESULtS}

Let $\mathbb{D}=\{z \in \mathbb{C}:|z|<1\}$ be the unit disk of the complex plane $\mathbb{C}$ and $\mathbb{T}=$ $\partial \mathbb{D}=\{\zeta \in \mathbb{C}:|\zeta|=1\}$ be the unit circle. The usual Lebesgue spaces for $\mathbb{T}$ are denoted by $L^{p}=L^{p}(\mathbb{T})$ and we write

$$
f(\zeta) \sim \sum_{n=-\infty}^{+\infty} \widehat{f}(n) \zeta^{n}
$$

for the Fourier series of a function $f$ in $L^{1}$. The Hardy spaces for $\mathbb{T}$ are defined by

$$
H^{p}=\left\{f \in L^{p}: \widehat{f}(n)=0 \text { for } n<0\right\}
$$

and their variants by $H_{0}^{p}=\left\{f \in L^{p}: \widehat{f}(n)=0\right.$ for $\left.n \leq 0\right\}$. We also define the spaces $\overline{H^{p}}=\left\{f \in L^{p}: \widehat{f}(n)=0\right.$ for $\left.n>0\right\}$ and the corresponding variants $\overline{H_{0}^{p}}=$ $\left\{f \in L^{p}: \widehat{f}(n)=0\right.$ for $\left.n \geq 0\right\}$.

The M. Riesz Theorem says that the Riesz projection $P$, defined by

$$
\operatorname{Pf}(\zeta) \sim \sum_{n=0}^{+\infty} \widehat{f}(n) \zeta^{n}
$$

for every $f(\zeta) \sim \sum_{n=-\infty}^{+\infty} \widehat{f}(n) \zeta^{n}$, is a bounded operator $L^{p} \rightarrow H^{p}$ when $1<p<\infty$; note, however, that the operator $P$ is not bounded either on $L^{1}$ or $L^{\infty}$. We also

1991 Mathematics Subject Classification. 47B35 (primary), 30D50, 30D55, 47A53 (secondary).

Key words and phrases. Hankel operators, Toeplitz operators, boundedness, compactness, Fredholmness, Hardy space $H^{1}$, bounded mean oscillation.

The first author was partially supported by the European Commission IHP Network "Harmonic Analysis and Related Problems" (Contract Number: HPRN-CT-2001-00273-HARP) and by the Greek Research Program "Pythagoras 2" (75\% European funds and 25\% National funds). The second author was fully supported by the European Commission IHP Network "Harmonic Analysis and Related Problems" (Contract Number: HPRN-CT-2001-00273-HARP) while he visited the first author at the University of Crete and later by the Academy of Finland Project 207048. 
define a related operator $P_{1}: L^{p} \rightarrow H_{0}^{p}$ by $P_{1} f(\zeta) \sim \sum_{n=1}^{+\infty} \widehat{f}(n) \zeta^{n}$ and denote the complementary projection of $P$ by $Q: L^{p} \rightarrow \overline{H_{0}^{p}}, Q f(\zeta) \sim \sum_{n=-\infty}^{-1} \widehat{f}(n) \zeta^{n}$. We say that $P f$ is the analytic part and $Q f$ is the antianalytic part of $f$.

The Toeplitz operator $T_{a}$ with symbol $a \in L^{2}$ is defined by

$$
T_{a} f=P(a f)
$$

and the Hankel operator $H_{a}$ by

$$
H_{a} f=P(a J f)
$$

where $J$ is the "flip operator" defined by

$$
J f(\zeta)=\bar{\zeta} f(\bar{\zeta}) \sim \sum_{n=-\infty}^{+\infty} \widehat{f}(-n-1) \zeta^{n} .
$$

Both operators $T_{a}$ and $H_{a}$ are obviously well defined for analytic polynomials, i.e. for finite sums $f(\zeta)=\sum_{n=0}^{N} \widehat{f}(n) \zeta^{n}$. The set of analytic polynomials is dense in each $H^{p}(1 \leq p<+\infty)$ and there are classical results which specify, for every particular value of $p$, the necessary and sufficient conditions on the symbol $a$ so that these operators are extended as bounded or even compact operators on $H^{p}$. It is easy to see that $T_{a}$ is not compact whenever $a$ is not the zero function. The situation is described by the following Theorems 1.1-1.5.

Theorem 1.1. Let $1<p<+\infty$. Then $T_{a}$ is bounded on $H^{p}$ if and only if $a \in L^{\infty}$.

Theorem 1.2. (Nehari, for $p=2$ ) Let $1<p<+\infty$. Then $H_{a}$ is bounded on $H^{p}$ if and only if $P_{1} a \in \mathrm{BMO}$.

Theorem 1.3. (Hartman, for $p=2$ ) Let $1<p<+\infty$. Then $H_{a}$ is compact on $H^{p}$ if and only if $P_{1} a \in \mathrm{VMO}$.

In the case of the space $H^{1}$ the results are slightly more complicated.

Theorem 1.4. (Stegenga, 1976, for real or antianalytic a; Janson-Peetre-Semmes, 1984; Tolokonnikov, 1987; Cima-Stegenga, 1987) The Toeplitz operator $T_{a}$ with symbol $a$ is bounded on $H^{1}$ if and only if $a \in L^{\infty}$ and $Q a \in \mathrm{BMO}_{\log }$.

Theorem 1.5. (Janson-Peetre-Semmes, 1984; Tolokonnikov, 1987; Cima-Stegenga, 1987) The Hankel operator $H_{a}$ is bounded on $H^{1}$ if and only if $P_{1} a \in \mathrm{BMO}_{\mathrm{log}}$.

The purpose of the first part of the article is to give a new proof of Theorem 1.5 together with a precise estimate of the operator norm of $H_{a}$ and to prove the analogous result about the compactness of $H_{a}$, that is, we prove the following two theorems.

Theorem 1.6. The Hankel operator $H_{a}$ is bounded on $H^{1}$ if and only if $P_{1} a \in$ $\mathrm{BMO}_{\log }$, in which case

$$
\left\|H_{a}\right\|_{H^{1} \rightarrow H^{1}} \asymp\left\|P_{1} a\right\|_{\mathrm{BMO}_{\log }},
$$

where $A \asymp B$ means that there are two positive numerical constants $c_{1}$ and $c_{2}$ so that $c_{1} \leq \frac{A}{B} \leq c_{2}$ for all values of the independent variables in $A$ and $B$.

Theorem 1.7. The Hankel operator $H_{a}$ is compact on $H^{1}$ if and only if $P_{1} a \in$ $\mathrm{VMO}_{\log }$. 
The second part of this article deals with spectral properties of Toeplitz operators. The case of continuous symbols was recently studied in [14]. Here we consider symbols that are not necessarily continuous. The motivation comes from the wellknown result on the Fredholm properties of Toeplitz operators on $H^{p}(1<p<\infty)$ with $a \in C+\overline{H^{\infty}}$, due to Douglas [6] when $p=2$. This suggests the following theorem, which is indeed the best we can hope for because of the differences in boundedness and compactness of the operators determined by the underlying spaces $H^{1}$ and $H^{p}$.

Theorem 1.8. Let $a \in \mathcal{V}+\overline{H^{\infty}} \cap \mathrm{BMO}_{\log }:=\mathcal{V}+\left(\overline{H^{\infty}} \cap \mathrm{BMO}_{\log }\right)$, where $\mathcal{V}=$ $C \cap \mathrm{VMO}_{\log }$. Then the following conditions are equivalent:

(1) $T_{a}$ is Fredholm on $H^{1}$, that is, $\operatorname{ker} T_{a}$ and coker $T_{a}$ are both of finite dimension;

(2) $a$ is invertible in the algebra $\mathcal{V}+\overline{H^{\infty}} \cap \mathrm{BMO}_{\log }$;

(3) $a$ is bounded away from zero, that is, there are $\epsilon>0$ and $\delta>0$ such that

$$
|a(z)| \geq \epsilon \text { for } 1-\delta<|z|<1,
$$

where $a(z)$ for $z \in \mathbb{D}$ is defined via the harmonic extension-see (1) below; in this case for any $1-\delta<r<1$

$$
\text { Ind } T_{a}:=\operatorname{dim} \operatorname{ker} T_{a}-\operatorname{dim} \operatorname{coker} T_{a}=-\operatorname{ind} a_{r}
$$

where $a_{r}(\zeta)=a(r \zeta)$ for all $\zeta \in \mathbb{T}$ and ind $a_{r}$ is the winding number of the function $a_{r}$.

\section{Preliminaries}

In this section we consider some (known) results from harmonic analysis. The Poisson extension of $f \in L^{1}$ at $z \in \mathbb{D}$ is given by

$$
f(z)=\frac{1}{2 \pi} \int_{\mathbb{T}} f(\zeta) \frac{1-|z|^{2}}{|\zeta-z|^{2}}|d \zeta|
$$

and the Szegö projection of $f$ at $z$ by

$$
P f(z)=\frac{1}{2 \pi i} \int_{\mathbb{T}} \frac{f(\zeta)}{\zeta-z} d \zeta .
$$

For $1 \leq p<+\infty$ and every $f \in L^{p}$ the limit $\lim _{r \rightarrow 1-} f(r \zeta)=f(\zeta)$ holds for almost every $\zeta \in \mathbb{T}$ and also in the $L^{p}$ sense. Since $f(z)$ is a harmonic function of $z \in \mathbb{D}$, it is also called the harmonic extension of $f$ in $\mathbb{D}$.

On the other hand, for $1 \leq p<+\infty$ and every $f \in L^{p}$, the limit

$$
\lim _{r \rightarrow 1-} \operatorname{Pf}(r \zeta)
$$

exists for almost every $\zeta \in \mathbb{T}$ and, when $1<p<+\infty$, this limit is equal to $\operatorname{Pf}(\zeta)$ (where $P$ is the Riesz projection) in both the almost everywhere sense and in the $L^{p}$ sense. In the case $p=1$, the limit $P f(\zeta)=\lim _{r \rightarrow 1-} P f(r \zeta)$ serves as the definition of the function $P f$ which, as is well known, belongs to the space $L^{1, w}$ of weak- $L^{1}$ functions. In all cases $\operatorname{Pf}(z)$ is an analytic function of $z \in \mathbb{D}$.

If $1<p<+\infty$ and $f \in L^{p}$, the Poisson extension of $P f \in L^{p}$ at every $z \in \mathbb{D}$ is equal to $\operatorname{Pf}(z)$ :

$$
\operatorname{Pf}(z)=\frac{1}{2 \pi} \int_{\mathbb{T}} \operatorname{Pf}(\zeta) \frac{1-|z|^{2}}{|\zeta-z|^{2}}|d \zeta|
$$


while if $1 \leq p<+\infty$ and $f \in H^{p}$, then (obviously) $\operatorname{Pf}(\zeta)=f(\zeta)$ and

$$
\operatorname{Pf}(z)=f(z)=\frac{1}{2 \pi} \int_{\mathbb{T}} f(\zeta) \frac{1-|z|^{2}}{|\zeta-z|^{2}}|d \zeta|=\frac{1}{2 \pi i} \int_{\mathbb{T}} \frac{f(\zeta)}{\zeta-z} d \zeta
$$

for every $z \in \mathbb{D}$.

We next consider the space of functions of bounded mean oscillation and its important (logarithmic) subspaces. A function $f$ is in BMO if $f \in L^{1}$ and

$$
\|f\|_{*}=\sup _{I} \frac{1}{|I|} \int_{I}\left|f(\zeta)-f_{I}\right||d \zeta|<+\infty,
$$

where the supremum is taken over all $\operatorname{arcs} I$ of $\mathbb{T}, f_{I}=\frac{1}{|I|} \int_{I} f(\zeta)|d \zeta|$ and $|I|$ is the length of $I$. The space BMO is a Banach space under the norm $\|f\|_{\text {BMO }}=$ $|\widehat{f}(0)|+\|f\|_{*}$. We also have the space BMOA of analytic functions in BMO, defined as $\mathrm{BMOA}=\mathrm{BMO} \cap H^{1}=\{f \in \mathrm{BMO}: \widehat{f}(n)=0$ for $n<0\}$.

It is well known that $L^{\infty} \subseteq \mathrm{BMO} \subseteq L^{p}$ for every $p<+\infty$ and that for every $f \in \mathrm{BMO}$

$$
\|f\|_{*} \asymp\left(\sup _{z \in \mathbb{D}} \int_{\mathbb{T}}|f(\zeta)-f(z)|^{2} \frac{1-|z|^{2}}{|\zeta-z|^{2}}|d \zeta|\right)^{\frac{1}{2}} .
$$

The subspace VMO of BMO contains by definition all functions $f \in L^{1}$ for which

$$
\lim _{\delta \rightarrow 0+} \sup _{I,|I|<\delta} \frac{1}{|I|} \int_{I}\left|f(\zeta)-f_{I}\right||d \zeta|=0 .
$$

We also define VMOA $=\mathrm{VMO} \cap H^{1}$. The space $\mathrm{VMO}$ is the closure in the space $\mathrm{BMO}$ of the set of all polynomials (or, equivalently, of all continuous functions). Also, $f \in$ BMO belongs to VMO if and only if $\lim _{r \rightarrow 1-}\left\|f_{r}-f\right\|_{\text {BMO }}=0$, where the function $f_{r}$ is defined by $f_{r}(\zeta)=f(r \zeta)$.

Somewhat less known are the spaces $\mathrm{BMO}_{\log }$ and $\mathrm{VMO}_{\log }$ and their variants $\mathrm{BMOA}_{\log }$ and $\mathrm{VMOA}_{\log }$. These are defined as follows. A function $f$ is in $\mathrm{BMO}_{\log }$ if $f \in L^{1}$ and

$$
\|f\|_{* *}=\sup _{I} \frac{\log \frac{4 \pi}{|I|}}{|I|} \int_{I}\left|f(\zeta)-f_{I}\right||d \zeta|<+\infty,
$$

where, again, the supremum is taken over all $\operatorname{arcs} I$ of $\mathbb{T}$. The space $\mathrm{BMO}_{\log }$ is a Banach space under the norm $\|f\|_{\mathrm{BMO}_{\log }}=|\widehat{f}(0)|+\|f\|_{* *}$. We define $\mathrm{BMOA}_{\log }=$ $\mathrm{BMO}_{\log } \cap H^{1}$.

It is obvious that $\mathrm{BMO}_{\log } \subseteq \mathrm{BMO}$. The following estimate

$$
\|f\|_{* *} \asymp\left(\sup _{z \in \mathbb{D}} \log ^{2} \frac{2}{1-|z|^{2}} \int_{\mathbb{T}}|f(\zeta)-f(z)|^{2} \frac{1-|z|^{2}}{|\zeta-z|^{2}}|d \zeta|\right)^{\frac{1}{2}}
$$

where $f \in \mathrm{BMO}_{\log }$, requires a similar consideration as in the case of the space $\mathrm{BMO}$, starting with the analogue of the John-Nirenberg theorem. The proofs do not seem to have been recorded anywhere but they are almost straightforward and, in any case, these facts have been used many times in the literature.

The logarithmic Lipschitz space Liplog is defined by

$$
\operatorname{Lip}_{\log }=\left\{f: \mathbb{T} \rightarrow \mathbb{C}: \sup _{\zeta, \eta \in \mathbb{T}} \log \frac{4}{|\zeta-\eta|}|f(\zeta)-f(\eta)|<\infty\right\} .
$$


This is a space of continuous functions under the norm

$$
\|f\|_{\text {Liplog }_{\text {log }}}=|\widehat{f}(0)|+\sup _{\zeta, \eta \in \mathbb{T}} \log \frac{4}{|\zeta-\eta|}|f(\zeta)-f(\eta)| .
$$

The space Liplog is continuously imbedded in $\mathrm{BMO}_{\log }$ and the main result of [9] is:

$$
\mathrm{BMO}_{\log }=\left\{f+P g: f, g \in \mathrm{Lip}_{\log }\right\} \text {. }
$$

In particular, if $h \in \mathrm{BMO}_{\log }$, there are $f, g \in \operatorname{Lip}_{\log }$ such that $h=f+P g$ and

$$
\|f\|_{\text {Liplog }_{\text {log }}}+\|g\|_{\text {Liplog }_{\text {log }}} \leq c\|h\|_{\mathrm{BMO}_{\log }} \text {. }
$$

where $c$ is a positive numerical constant.

The subspace $\mathrm{VMO}_{\log }$ of $\mathrm{BMO}_{\log }$ contains by definition all functions $f \in L^{1}$ for which

$$
\lim _{\delta \rightarrow 0+} \sup _{I,|I|<\delta} \frac{\log \frac{4 \pi}{|I|}}{|I|} \int_{I}\left|f(\zeta)-f_{I}\right||d \zeta|=0 .
$$

We also define $\mathrm{VMOA}_{\log }=\mathrm{VMO}_{\log } \cap H^{1}$. The following two results will be needed several times.

Theorem 2.1. For the logarithmic VMO space, we have the following characterization:

$$
\mathrm{VMO}_{\log }=\{f+P g: f, g \in \operatorname{lip} \log \},
$$

where liplog stands for the so-called vanishing logarithmic Lipschitz space defined by

$$
\operatorname{lip}_{\log }=\left\{f \in \operatorname{Lip}_{\log }: \lim _{\delta \rightarrow 0+} \sup _{|\zeta-\eta|<\delta} \log \frac{4}{|\zeta-\eta|}|f(\zeta)-f(\eta)|=0\right\} .
$$

Theorem 2.2. For $f \in \mathrm{BMO}_{\mathrm{log}}$, the following conditions are equivalent:

(1) $f \in \mathrm{VMO}_{\text {log }}$;

(2) $\lim _{\eta \rightarrow 1}\left\|\tau_{\eta} f-f\right\|_{\mathrm{BMO}_{\log }}=0$, where $\tau_{\eta} f(\zeta)=f(\zeta \bar{\eta})$ for $\eta, \zeta \in \mathbb{T}$;

(3) $\lim _{r \rightarrow 1-}\left\|f_{r}-f\right\|_{\mathrm{BMO}_{\log }}=0$, where $f_{r}(\zeta)=f(r \zeta)$ for $\zeta \in \mathbb{T}$.

The following descriptions are also useful:

$$
\left\{a \in L^{\infty}: Q a \in \mathrm{BMO}_{\log }\right\}=\operatorname{Lip}_{\log }+H^{\infty}
$$

and

$$
\left\{a \in L^{\infty}: Q a \in \mathrm{VMO}_{\log }\right\}=\operatorname{lip} \log +H^{\infty} .
$$

These can be verified by means of the characterizations in (4) and (5); for example, if $a=l+h \in \operatorname{lip} \log +H^{\infty}$, then $a \in L^{\infty}$ and $Q a=Q l \in \mathrm{VMO}_{\log }$, and conversely if $a \in L^{\infty}$ and $Q a \in \mathrm{VMO}_{\log }$, then $Q a=f+P g$ for some $f, g \in \operatorname{lip}$ log, so $Q a=Q f$, which implies that $a-f \in H^{\infty}$ and we can write $a=f+(a-f) \in \operatorname{lip} \log +H^{\infty}$.

For each arc $I$ we define $S(I)=\left\{z \in \mathbb{D}: 0<1-|z|<\frac{|I|}{2 \pi}, \frac{z}{|z|} \in I\right\}$, called the Carleson "square" with base $I$. A positive Borel measure $\mu$ in $\mathbb{D}$ is called a Carleson measure if

$$
\sup _{I} \frac{\mu(S(I))}{|I|}<+\infty
$$

where the supremum is taken over all $\operatorname{arcs} I$ of $\mathbb{T}$. It is known that $\mu$ is a Carleson measure if and only if there is a constant $c$ so that

$$
\iint_{\mathbb{D}}|f(z)|^{2} d \mu(z) \leq c \int_{\mathbb{T}}|f(\zeta)|^{2}|d \zeta|, \quad f \in L^{2},
$$


and that, if $c$ is the smallest constant for which this inequality holds,

$$
c \asymp \sup _{I} \frac{\mu(S(I))}{|I|} .
$$

In this connection, we have a function $f \in L^{1}$ in BMO if and only if the Borel measure $|\nabla f(z)|^{2}\left(1-|z|^{2}\right) d m(z)$, where $d m$ is the area measure, is a Carleson measure and

$$
\|f\|_{*} \asymp\left(\sup _{I} \frac{1}{|I|} \iint_{S(I)}|\nabla f(z)|^{2}\left(1-|z|^{2}\right) d m(z)\right)^{\frac{1}{2}} .
$$

Similarly, $f \in L^{1}$ is in VMO if and only if

$$
\lim _{\delta \rightarrow 0+} \sup _{I,|I|<\delta} \frac{1}{|I|} \iint_{S(I)}|\nabla f(z)|^{2}\left(1-|z|^{2}\right) d m(z)=0 .
$$

Of course, in the case of $f \in H^{1}$ we may replace $\nabla f(z)$ by $f^{\prime}(z)$ in the above characterizations of BMO and VMO.

Analogously, for functions $f$ in $\mathrm{BMO}_{\log }$, we have

$$
\|f\|_{* *} \asymp\left(\sup _{I} \frac{\log ^{2} \frac{4 \pi}{|I|}}{|I|} \iint_{S(I)}|\nabla f(z)|^{2}\left(1-|z|^{2}\right) d m(z)\right)^{\frac{1}{2}} .
$$

Note also that there exists a positive numerical constant $c$ so that for every $f \in$ BMOA and every $z \in \mathbb{D}$ :

$$
|f(z)| \leq c\|f\|_{\mathrm{BMO}} \log \frac{2}{1-|z|^{2}} .
$$

Conversely, there exists a positive numerical constant $c$ so that for every $z \in \mathbb{D}$ there exists an $f \in$ BMOA with

$$
\|f\|_{\mathrm{BMO}}=1, \quad|f(z)| \geq c \log \frac{2}{1-|z|^{2}} .
$$

Also, if $f \in \mathrm{VMOA}$ then

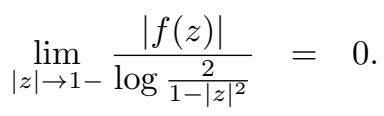

Finally, we shall use the inequality

$$
|\langle f, g\rangle| \leq c\|f\|_{H^{1}}\|g\|_{\mathrm{BMOA}},
$$

where the binary form $\langle\cdot, \cdot\rangle$ is defined by

$$
\langle f, g\rangle=\lim _{r \rightarrow 1-} \frac{1}{2 \pi} \int_{\mathbb{T}} f_{r}(\zeta) g(\bar{\zeta})|d \zeta|=\lim _{r \rightarrow 1-} \frac{1}{2 \pi i} \int_{\mathbb{T}} f(r \zeta) g(\bar{\zeta}) \bar{\zeta} d \zeta .
$$

The Fefferman-Stein duality which is induced by this binary form says that BMOA is isomorphic to $\left(H^{1}\right)^{*}$. It is also true that, under the same binary form, $H^{1}$ is isomorphic to (VMOA)*. 


\section{Proof of Theorem 1.6}

Proof. Before proceeding to the proof, note that the part $a-P_{1} a$ of $a$ plays no role in the Hankel operator $H_{a}$. Indeed, for all analytic polynomials $f$ the function $\left(a-P_{1} a\right) J f$ is antianalytic and, hence, $H_{a} f=H_{P_{1} a} f$. We may thus suppose in all that follows that $a=P_{1} a$ or in other words that

$$
\widehat{a}(n)=0, \quad n \leq 0 .
$$

We recall that BMOA is isomorphic to the dual space of $H^{1}$ and it is easy to see that, formally at least, the dual operator to $H_{a}$ on $H^{1}$ is $H_{a}$ on BMOA. This means that

$$
\left\langle H_{a} f, g\right\rangle=\left\langle f, H_{a} g\right\rangle
$$

for all analytic polynomials $f$ and all $g \in$ BMOA. Hence, we need to prove that $H_{a}$ is bounded on BMOA if and only if $a \in \mathrm{BMO}_{\log }$ and that

$$
\left\|H_{a}\right\|_{\mathrm{BMOA} \rightarrow \mathrm{BMOA}} \asymp\|a\|_{\mathrm{BMO}_{\mathrm{log}}}
$$

under the assumption (16).

Sufficiency. Let $a \in \mathrm{BMO}_{\log }$ satisfy (16) and take an arbitrary $f \in \mathrm{BMOA}$. Then

$$
H_{a} f(z)=\frac{1}{2 \pi i} \int_{\mathbb{T}} \frac{a(\zeta) \bar{\zeta} f(\bar{\zeta})}{\zeta-z} d \zeta=\frac{1}{2 \pi i} \int_{\mathbb{T}} \frac{b(\zeta) \overline{g(\zeta)}}{\zeta-z} d \zeta
$$

where we set $b(\zeta)=\bar{\zeta} a(\zeta)$ and $g(\zeta)=\overline{f(\bar{\zeta})}$. It is obvious that $b \in \mathrm{BMOA}_{\log }$ with $\|b\|_{\mathrm{BMO}_{\mathrm{log}}} \asymp\|a\|_{\mathrm{BMO}_{\mathrm{log}}}$ and that $g \in \mathrm{BMOA}$ with $\|g\|_{\mathrm{BMO}}=\|f\|_{\mathrm{BMO}}$.

Throughout, the symbol $c$ denotes a numerical constant, not necessarily the same at each occurrence. We have

$$
\begin{aligned}
\left(H_{a} f\right)^{\prime}(z) & =\frac{1}{2 \pi i} \int_{\mathbb{T}} \frac{b(\zeta) \overline{g(\zeta)}}{(\zeta-z)^{2}} d \zeta \\
& =\frac{1}{2 \pi i} \int_{\mathbb{T}} \frac{b(\zeta) \overline{(g(\zeta)-g(z))}}{(\zeta-z)^{2}} d \zeta+\overline{g(z)} \frac{1}{2 \pi i} \int_{\mathbb{T}} \frac{b(\zeta)}{(\zeta-z)^{2}} d \zeta \\
& =\frac{1}{2 \pi i} \int_{\mathbb{T}} \frac{(b(\zeta)-b(z) \overline{(g(\zeta)-g(z))}}{(\zeta-z)^{2}} d \zeta+\overline{g(z)} b^{\prime}(z) .
\end{aligned}
$$

Applying the Cauchy-Schwarz inequality together with (2) and (3), we get

$$
\begin{aligned}
\left|\left(H_{a} f\right)^{\prime}(z)\right|^{2} & \leq c \int_{\mathbb{T}} \frac{|b(\zeta)-b(z)|^{2}}{|\zeta-z|^{2}}|d \zeta| \int_{\mathbb{T}} \frac{|g(\zeta)-g(z)|^{2}}{|\zeta-z|^{2}}|d \zeta|+c|g(z)|^{2}\left|b^{\prime}(z)\right|^{2} \\
& \leq c\|b\|_{* *}^{2}\|g\|_{*}^{2} \frac{1}{\left(1-|z|^{2}\right)^{2} \log ^{2} \frac{2}{1-|z|^{2}}}+c|g(z)|^{2}\left|b^{\prime}(z)\right|^{2}
\end{aligned}
$$


This, for every $\operatorname{arc} I$ of $\mathbb{T}$, implies

$$
\begin{aligned}
& \frac{1}{|I|} \iint_{S(I)}\left|\left(H_{a} f\right)^{\prime}(z)\right|^{2}\left(1-|z|^{2}\right) d m(z) \\
& \leq c\|b\|_{* *}^{2}\|g\|_{*}^{2} \frac{1}{|I|} \iint_{S(I)} \frac{1}{\left(1-|z|^{2}\right) \log ^{2} \frac{2}{1-|z|^{2}}} d m(z) \\
& +c \frac{1}{|I|} \iint_{S(I)}\left|g(z)-g\left(z_{I}\right)\right|^{2}\left|b^{\prime}(z)\right|^{2}\left(1-|z|^{2}\right) d m(z) \\
& +c\left|g\left(z_{I}\right)\right|^{2} \frac{1}{|I|} \iint_{S(I)}\left|b^{\prime}(z)\right|^{2}\left(1-|z|^{2}\right) d m(z) \\
& =A+B+C
\end{aligned}
$$

where $z_{I}$ is the point in the middle of the internal side of $S(I)$, defined by $1-\left|z_{I}\right|=$ $\frac{|I|}{2 \pi}$ and $\frac{z_{I}}{\left|z_{I}\right|}=$ midpoint of $I$.

Let us first estimate the term $A$. A direct calculation of the integral, using polar coordinates, gives

$$
\begin{aligned}
& A \leq c \frac{1}{\log \frac{4 \pi}{|I|}}\|b\|_{* *}^{2}\|g\|_{*}^{2} \\
& \leq \quad c \frac{1}{\log \frac{4 \pi}{|I|}}\|a\|_{\mathrm{BMO}_{\log }}^{2}\|f\|_{\mathrm{BMO}}^{2} .
\end{aligned}
$$

Observing that $\left|1-\overline{z_{I}} z\right| \asymp|I|$ for all $z \in S(I)$ and considering the Borel measure $d \mu(z)$ which is equal to $\left|b^{\prime}(z)\right|^{2}\left(1-|z|^{2}\right) d m(z)$ on $S(I)$ and equal to zero on $\mathbb{D} \backslash S(I)$, we find using (8) and (9) that

$$
\begin{aligned}
B & \leq c|I| \iint_{S(I)} \frac{\left|g(z)-g\left(z_{I}\right)\right|^{2}}{\left|1-\overline{z_{I}} z\right|^{2}}\left|b^{\prime}(z)\right|^{2}\left(1-|z|^{2}\right) d m(z) \\
& \leq c|I| \sup _{J} \frac{\mu(S(J))}{|J|} \int_{\mathbb{T}} \frac{\left|g(\zeta)-g\left(z_{I}\right)\right|^{2}}{\left|1-\overline{z_{I}} \zeta\right|^{2}}|d \zeta| \\
& \leq c \sup _{J} \frac{\mu(S(J))}{|J|} \int_{\mathbb{T}}\left|g(\zeta)-g\left(z_{I}\right)\right|^{2} \frac{1-\left|z_{I}\right|^{2}}{\left|\zeta-z_{I}\right|^{2}}|d \zeta| \\
& \leq c\|g\|_{*}^{2} \sup _{J} \frac{\mu(S(J))}{|J|} .
\end{aligned}
$$

Estimating $\frac{\mu(S(J))}{|J|}=\frac{\mu(S(J) \cap S(I))}{|J|}$, we observe that we need only consider arcs $J$ having nonempty intersection with $I$. In the case $|J|>|I|, \frac{\mu(S(J))}{|J|} \leq \frac{\mu(S(I))}{|I|}$. If $|J| \leq|I|$, then $J \subseteq 3 I$, where $3 I$ is the arc with the same midpoint as $I$ and with length three times the length of $I$. Hence, in both cases we get using (12)

$$
\begin{aligned}
\sup _{J} \frac{\mu(S(J))}{|J|} & \leq \sup _{J \subseteq 3 I} \frac{1}{|J|} \iint_{S(J)}\left|b^{\prime}(z)\right|^{2}\left(1-|z|^{2}\right) d m(z) \\
& \leq c \sup _{J \subseteq 3 I} \frac{1}{\log ^{2} \frac{4 \pi}{|J|}}\|b\|_{* *}^{2} \\
& \leq c \frac{1}{\log ^{2} \frac{4 \pi}{|I|}\|b\|_{* *}^{2} .}
\end{aligned}
$$


Therefore (19) implies

$$
\begin{aligned}
B & \leq c \frac{1}{\log ^{2} \frac{4 \pi}{|I|}}\|b\|_{* *}^{2}\|g\|_{*}^{2} \\
& \leq c \frac{1}{\log ^{2} \frac{4 \pi}{|I|}}\|a\|_{\mathrm{BMO}_{\log }}^{2}\|f\|_{\mathrm{BMO}}^{2} .
\end{aligned}
$$

Finally, (12) and (13) imply

$$
\begin{aligned}
C & \leq c\|b\|_{* *}^{2}\|g\|_{\mathrm{BMO}}^{2} \\
& \leq c\|a\|_{\mathrm{BMO}_{\log }}^{2}\|f\|_{\mathrm{BMO}}^{2} .
\end{aligned}
$$

Now, estimates (18), (20) and (21) together with (17) imply

$$
\frac{1}{|I|} \iint_{S(I)}\left|\left(H_{a} f\right)^{\prime}(z)\right|^{2}\left(1-|z|^{2}\right) d m(z) \leq c\|a\|_{\mathrm{BMO} \log }^{2}\|f\|_{\mathrm{BMO}}^{2}
$$

and, taking the supremum over all arcs $I$ and using (10),

$$
\left\|H_{a} f\right\|_{*} \leq c\|a\|_{\mathrm{BMO}_{\log }}\|f\|_{\mathrm{BMO}} .
$$

On the other hand,

$$
\begin{aligned}
\left|\widehat{H_{a} f}(0)\right|^{2} & \leq c \int_{\mathbb{T}}\left|H_{a} f(\zeta)\right|^{2}|d \zeta|=c \int_{\mathbb{T}}|a(\zeta)|^{2}|f(\zeta)|^{2}|d \zeta| \\
& \leq c\left(\int_{\mathbb{T}}|a(\zeta)|^{4}|d \zeta|\right)^{\frac{1}{2}}\left(\int_{\mathbb{T}}|f(\zeta)|^{4}|d \zeta|\right)^{\frac{1}{2}} \\
& \leq c\|a\|_{\mathrm{BMO}}^{2}\|f\|_{\mathrm{BMO}}^{2} \leq c\|a\|_{\mathrm{BMO}_{\log }}^{2}\|f\|_{\mathrm{BMO}}^{2} .
\end{aligned}
$$

The last two estimates show that

$$
\left\|H_{a} f\right\|_{\mathrm{BMO}} \leq c\|a\|_{\mathrm{BMO}_{\log }}\|f\|_{\mathrm{BMO}}
$$

and hence

$$
\left\|H_{a}\right\|_{\mathrm{BMOA} \rightarrow \mathrm{BMOA}} \leq c\|a\|_{\mathrm{BMO}_{\mathrm{log}}} .
$$

Necessity, step 1 . Here we make the a priori assumption that $a \in B M O_{\log }$ (and, that $a$ satisfies (16)) and we set $b(\zeta)=\bar{\zeta} a(\zeta)$ as before.

$$
\text { If }|\widehat{b}(0)| \geq \frac{1}{2}\|b\|_{B M O_{\log }} \text {, then }
$$

$$
\begin{aligned}
\left\|H_{a}\right\|_{B M O A \rightarrow B M O A} & \geq\left\|H_{a} 1\right\|_{B M O}=\|b\|_{B M O} \\
& \geq \widehat{b}(0) \mid \geq \frac{1}{2}\|b\|_{B M O_{\log }} \\
& \geq c\|a\|_{B M O_{\log }} .
\end{aligned}
$$

If $|\widehat{b}(0)|<\frac{1}{2}\|b\|_{B M O_{\log }}$, then $\|b\|_{* *} \geq \frac{1}{2}\|b\|_{B M O_{\log }}$ and based on (12) we find an arc $I$ such that

$$
c\|a\|_{B M O_{\log }}^{2} \leq c\|b\|_{B M O_{\log }}^{2} \leq \frac{\log ^{2} \frac{4 \pi}{|I|}}{|I|} \iint_{S(I)}\left|b^{\prime}(z)\right|^{2}\left(1-|z|^{2}\right) d m(z) .
$$

Through (14) we find an $f \in \mathrm{BMOA}$ and the corresponding $g(\zeta)=\overline{f(\bar{\zeta})}$ so that

$$
\|f\|_{\mathrm{BMO}}=1, \quad c \log \frac{4 \pi}{|I|} \leq\left|f\left(\overline{z_{I}}\right)\right|=\left|g\left(z_{I}\right)\right| .
$$


The trivial variant of inequality (17) together with the estimates (18), (20), (22) and (23) imply

$$
\begin{aligned}
\left\|H_{a}\right\|_{\mathrm{BMOA} \rightarrow \mathrm{BMOA}}^{2} & \geq\left\|H_{a} f\right\|_{\mathrm{BMO}}^{2} \\
& \geq \frac{1}{|I|} \iint_{S(I)}\left|\left(H_{a} f\right)^{\prime}(z)\right|^{2}\left(1-|z|^{2}\right) d m(z) \\
& \geq C-A-B \geq c\left(1-c \frac{1}{\log \frac{4 \pi}{|I|}}\right)\|a\|_{\mathrm{BMO}_{\log }}^{2} .
\end{aligned}
$$

Hence, if $|I|$ is smaller than a certain positive numerical constant we find that

$$
\left\|H_{a}\right\|_{\mathrm{BMOA} \rightarrow \mathrm{BMOA}} \geq c\|a\|_{\mathrm{BMO}_{\mathrm{log}}} \text {. }
$$

On the other hand, if $|I|$ is larger than the same positive numerical constant, then

$$
\begin{aligned}
\left\|H_{a}\right\|_{\mathrm{BMOA} \rightarrow \mathrm{BMOA}}^{2} & \geq\left\|H_{a} 1\right\|_{\mathrm{BMO}}^{2}=\|b\|_{\mathrm{BMO}}^{2} \\
& \geq c \frac{1}{|I|} \iint_{S(I)}\left|b^{\prime}(z)\right|^{2}\left(1-|z|^{2}\right) d m(z) \\
& \geq c \frac{\log ^{2} \frac{4 \pi}{|I|}}{|I|} \iint_{S(I)}\left|b^{\prime}(z)\right|^{2}\left(1-|z|^{2}\right) d m(z) \\
& \geq c\|a\|_{\mathrm{BMO}_{\log }}^{2} .
\end{aligned}
$$

We conclude that if $a$ is assumed to be in $\mathrm{BMO}_{\log }$ and satisfy (16) then

$$
\left\|H_{a}\right\|_{\mathrm{BMOA} \rightarrow \mathrm{BMOA}} \geq c\|a\|_{\mathrm{BMO}_{\log }}
$$

and, by the usual duality,

$$
\left\|H_{a}\right\|_{H^{1} \rightarrow H^{1}} \geq c\|a\|_{\mathrm{BMO}_{\log }} .
$$

Lemma 3.1. If $H_{a}$ is bounded on $H^{1}$, then for every $f \in H^{1}$ and all $r<1$ we have $H_{a_{r}} f=r\left(H_{a} f_{r}\right)_{r}$.

Proof. The operator $H_{a_{r}}$ is bounded on $H^{1}$ since $a_{r}$ is smooth. Verifying the equality involves a straightforward calculation using Fourier series.

Necessity, step 2. Applying the a priori estimate of step 1 to the functions $a_{r}$ we have

$$
\left\|H_{a_{r}}\right\|_{H^{1} \rightarrow H^{1}} \geq c\left\|a_{r}\right\|_{\mathrm{BMO}_{\log }} .
$$

The lemma of Fatou with (12) implies that

$$
\|a\|_{\mathrm{BMO}_{\log }} \leq \liminf _{r \rightarrow 1-}\left\|a_{r}\right\|_{\mathrm{BMO}_{\log }} .
$$

Now, Lemma 3.1 implies that for all $f \in H^{1}$

$$
\begin{aligned}
\left\|H_{a_{r}} f\right\|_{H^{1}} & \leq r\left\|H_{a} f_{r}\right\|_{H^{1}} \\
& \leq\left\|H_{a}\right\|_{H^{1} \rightarrow H^{1}}\left\|f_{r}\right\|_{H^{1}} \\
& \leq\left\|H_{a}\right\|_{H^{1} \rightarrow H^{1}}\|f\|_{H^{1}}
\end{aligned}
$$

and hence

$$
\left\|H_{a_{r}}\right\|_{H^{1} \rightarrow H^{1}} \leq\left\|H_{a}\right\|_{H^{1} \rightarrow H^{1}} .
$$

Relations (24), (25) and (26) complete the necessity part of Theorem 1.6. 


\section{Proof of Theorem 1.7}

Proof. Let $a \in \mathrm{VMO}_{\log }$ satisfy (16). Let $r<1$ and take $f_{n} \in H^{1}$ with $\left\|f_{n}\right\|_{H^{1}} \leq 1$. Choosing a subsequence, we may assume that there is a function $f \in H^{1}$ so that $\left(f_{n}\right)_{r} \rightarrow f$ in $H^{1}$. Since $H_{a}$ is bounded, we get $H_{a}\left(f_{n}\right)_{r} \rightarrow H_{a} f$ in $H^{1}$ and, hence, $H_{a_{r}} f_{n}=r\left(H_{a}\left(f_{n}\right)_{r}\right)_{r} \rightarrow r\left(H_{a} f\right)_{r}$ in $H^{1}$. Therefore, $H_{a_{r}}$ is compact on $H^{1}$. Finally, $\left\|H_{a_{r}}-H_{a}\right\|_{H^{1} \rightarrow H^{1}} \leq c\left\|a_{r}-a\right\|_{\mathrm{BMO}_{\log }} \rightarrow 0$ as $r \rightarrow 1-$ and, hence, $H_{a}$ is compact on $H^{1}$.

Let $a \in \mathrm{BMO}_{\log }$ satisfy (16). It is a consequence of the proof of Theorem 1.6 that $H_{a}$ is bounded on VMOA. Indeed, taking any $f \in \mathrm{VMOA}$, (17) together with (12), (15), (18) and (20) imply that

$$
\begin{aligned}
& \sup _{I,|I|<\delta} \frac{1}{|I|} \iint_{S(I)}\left|\left(H_{a} f\right)^{\prime}(z)\right|^{2}\left(1-|z|^{2}\right) d m(z) \\
& \leq c \frac{1}{\log \frac{4 \pi}{\delta}}\|a\|_{\mathrm{BMO}_{\log }}^{2}\|f\|_{\mathrm{BMO}}^{2}+c\|a\|_{\mathrm{BMO}_{\log }^{2}}^{2} \sup _{I,|I|<\delta} \frac{\left|f\left(z_{I}\right)\right|^{2}}{\log ^{2} \frac{4 \pi}{|I|}} \\
& \rightarrow 0
\end{aligned}
$$

as $\delta \rightarrow 0+$. Therefore, (11) implies that $H_{a} f \in \mathrm{VMOA}$.

Now, if we assume that $H_{a}$ is compact on $H^{1}$ then it is also compact on VMOA, since $H^{1}$ is isomorphic to (VMOA)* . To get a contradiction we suppose that $a$ does not belong to $\mathrm{VMO}_{\log }$. Then there exist some $\delta>0$ and $r_{n} \rightarrow 1$ - such that

$$
\left\|a_{r_{n}}-a\right\|_{\mathrm{BMO}_{\log }} \geq \delta \text {. }
$$

This implies

$$
\left\|H_{a_{r_{n}}}-H_{a}\right\|_{\mathrm{BMO}_{\log }} \geq c \delta
$$

and we can choose $f_{n} \in H^{1}$ with $\left\|f_{n}\right\|_{H^{1}} \leq 1$, so that

$$
\begin{aligned}
c \delta & \leq\left\|H_{a_{r_{n}}} f_{n}-H_{a} f_{n}\right\|_{H^{1}} \\
& =\left\|r_{n}\left(H_{a}\left(f_{n}\right)_{r_{n}}\right)_{r_{n}}-H_{a} f_{n}\right\|_{H^{1}} \\
& =\left\|\left(H_{a}\left(f_{n}\right)_{r_{n}}\right)_{r_{n}}-H_{a} f_{n}\right\|_{H^{1}}+o(1) \\
& \leq\left\|\left(H_{a}\left(f_{n}\right)_{r_{n}}\right)_{r_{n}}-\left(H_{a} f_{n}\right)_{r_{n}}\right\|_{H^{1}}+\left\|\left(H_{a} f_{n}\right)_{r_{n}}-H_{a} f_{n}\right\|_{H^{1}}+o(1) \\
& \leq\left\|H_{a}\left(f_{n}\right)_{r_{n}}-H_{a} f_{n}\right\|_{H^{1}}+\left\|\left(H_{a} f_{n}\right)_{r_{n}}-H_{a} f_{n}\right\|_{H^{1}}+o(1) .
\end{aligned}
$$

Taking a subsequence, if necessary, we may assume that there is a $v \in H^{1}$ so that $H_{a} f_{n} \rightarrow v$ in $H^{1}$. Therefore

$$
\begin{aligned}
c \delta \leq & \left\|H_{a}\left(\left(f_{n}\right)_{r_{n}}-f_{n}\right)\right\|_{H^{1}}+\left\|\left(H_{a} f_{n}\right)_{r_{n}}-v_{r_{n}}\right\|_{H^{1}}+\left\|v_{r_{n}}-v\right\|_{H^{1}} \\
& +\left\|v-H_{a} f_{n}\right\|_{H^{1}}+o(1) \\
= & \left\|H_{a}\left(\left(f_{n}\right)_{r_{n}}-f_{n}\right)\right\|_{H^{1}}+o(1) .
\end{aligned}
$$

If we choose $h_{n} \in \mathrm{VMOA}$ with $\left\|h_{n}\right\|_{\mathrm{BMOA}}=1$ and

$$
c\left\|H_{a}\left(\left(f_{n}\right)_{r_{n}}-f_{n}\right)\right\|_{H^{1}} \leq\left\langle H_{a}\left(\left(f_{n}\right)_{r_{n}}-f_{n}\right), h_{n}\right\rangle,
$$

we have

$$
\begin{aligned}
c \delta & \leq\left\langle H_{a}\left(\left(f_{n}\right)_{r_{n}}-f_{n}\right), h_{n}\right\rangle+o(1) \\
& =\left\langle\left(f_{n}\right)_{r_{n}}-f_{n}, H_{a} h_{n}\right\rangle+o(1) \\
& =\left\langle f_{n},\left(H_{a} h_{n}\right)_{r_{n}}-H_{a} h_{n}\right\rangle+o(1) .
\end{aligned}
$$


Since $H_{a}$ is compact on VMOA, taking a subsequence once more we see that there is a $w \in \mathrm{VMOA}$ so that $H_{a} h_{n} \rightarrow w$ in VMOA. Hence

$$
\begin{aligned}
c \delta & \leq\left\langle f_{n},\left(H_{a} h_{n}\right)_{r_{n}}-w_{r_{n}}\right\rangle+\left\langle f_{n}, w_{r_{n}}-w\right\rangle+\left\langle f_{n}, w-H_{a} h_{n}\right\rangle+o(1) \\
& \leq c\left\|\left(H_{a} h_{n}\right)_{r_{n}}-w_{r_{n}}\right\|_{\mathrm{BMO}}+c\left\|w_{r_{n}}-w\right\|_{\mathrm{BMO}}+c\left\|w-H_{a} h_{n}\right\|_{\mathrm{BMO}}+o(1) \\
& \leq c\left\|w_{r_{n}}-w\right\|_{\mathrm{BMO}}+o(1) \\
& =o(1) .
\end{aligned}
$$

This is false and hence $a \in \mathrm{VMO}_{\log }$.

\section{Fredholmness of Toeplitz operators}

We start by proving the equivalence of the criteria (2) and (3) for Fredholmness in Theorem 1.8. We use the symbol $\chi_{n}$ for the functions

$$
\chi_{n}(\zeta)=\zeta^{n}, \quad \zeta \in \mathbb{T} .
$$

Lemma 5.1. The functions in $\mathcal{V}+\overline{H^{\infty}} \cap \mathrm{BMO}_{\log }$ can be approximated in the space $L^{\infty} \cap \mathrm{BMO}_{\log }$ by functions of the form $\chi_{n} h$ with $n \geq 0$ and $h \in \overline{H^{\infty}} \cap \mathrm{BMO}_{\log }$.

Proof. Let $v+b \in \mathcal{V}+\overline{H^{\infty}} \cap \mathrm{BMO}_{\log }$. According to (3) of Theorem 2.2, there are trigonometric polynomials $p_{k}$ such that $\left\|v-p_{k}\right\|_{L^{\infty} \cap \mathrm{BMO}_{\mathrm{log}}} \rightarrow 0$. Since

$$
p_{k}+b \in\left\{\chi_{n} h: n \geq 0, h \in \overline{H^{\infty}} \cap \mathrm{BMO}_{\log }\right\},
$$

the proof is complete.

Proposition 5.2. Let $a \in \mathcal{V}+\overline{H^{\infty}} \cap \mathrm{BMO}_{\log }$. Then a is invertible in $\mathcal{V}+\overline{H^{\infty}} \cap$ $\mathrm{BMO}_{\log }$ if and only if $a$ is bounded away from zero, that is, there are $\epsilon>0$ and $\delta>0$ such that

$$
|a(z)| \geq \epsilon \quad \text { for } 1-\delta<|z|<1 .
$$

Proof. If $a$ is invertible in $\mathcal{V}+\overline{H^{\infty}} \cap \mathrm{BMO}_{\log }$, then it is obviously invertible in $C+\overline{H^{\infty}}$ and thus bounded away from zero according to [7, Theorem 6.45].

By the preceding lemma, there are $N \rightarrow+\infty$ and corresponding $h_{N} \in \overline{H^{\infty}} \cap$ $\mathrm{BMO}_{\log }$ such that $\left\|a-\chi_{N} h_{N}\right\|_{L^{\infty} \cap \mathrm{BMO}_{\log }} \rightarrow 0$. By [7, Theorem 6.45], $h_{N}$ is invertible in $\overline{H^{\infty}}$ with $N$ sufficiently large. As $h_{N} \in \mathrm{BMO}_{\text {log }}$, so is its inverse. Thus, $\chi_{N} h_{N}$ is invertible in $\mathcal{V}+\overline{H^{\infty}} \cap \mathrm{BMO}_{\log }$. Now $\chi_{-N} h_{N}{ }^{-1} \rightarrow a^{-1}$ in $L^{\infty} \cap \mathrm{BMO}_{\log }$ and so $a^{-1}$ is in the closed space $\mathcal{V}+\overline{H^{\infty}} \cap \mathrm{BMO}_{\log }$.

It remains to show that the two conditions above are indeed sufficent and necessary for Fredholmness. This follows from Theorem 5.6 and Proposition 5.9 below.

Let us first consider two basic results for quite general symbols that are needed in what follows.

Proposition 5.3. Let $a, b \in L^{\infty} \cap \mathrm{BMO}_{\log }$. Then

$$
\begin{aligned}
T_{a b} & =T_{a} T_{b}+H_{a} H_{\tilde{b}}, \\
H_{a b} & =T_{a} H_{b}+H_{a} T_{\tilde{b}},
\end{aligned}
$$

where $\tilde{b}(\zeta)=b(1 / \zeta), \zeta \in \mathbb{T}$.

Proof. See, e.g., [3, Proposition 2.14].

The next theorem gives a necessary condition for Fredholmness - cf. the wellknown theorem of Simonenko in the case of $1<p<\infty$. 
Theorem 5.4. Let $a \in L^{\infty} \cap \mathrm{BMO}_{\log }$. If $T_{a}$ is Fredholm, then

$$
\operatorname{ess~} \inf _{\zeta \in \mathbb{T}}|a(\zeta)|>0
$$

in particular, the symbol is invertible in $L^{\infty} \cap \mathrm{BMO}_{\log }$.

Proof. Suppose that $T_{a}$ is Fredholm but ess $\inf _{\zeta \in \mathbb{T}}|a(\zeta)|=0$.

We consider a small $\epsilon>0$ and decompose $a=u+i v$ into real and imaginary parts. Define $u_{\epsilon}=\max (u, \epsilon)+\min (u,-\epsilon)$ and $v_{\epsilon}$ by the analogous formula. Now the function $a_{\epsilon}=u_{\epsilon}+i v_{\epsilon}$ is equal to 0 on a set of positive measure and $\| a-$ $a_{\epsilon} \|_{L^{\infty} \cap B M O_{\log }} \rightarrow 0$ as $\epsilon \rightarrow 0+$. This implies that $\left\|T_{a}-T_{a_{\epsilon}}\right\| \rightarrow 0$ as $\epsilon \rightarrow 0+$ and, hence, that $T_{a_{\epsilon}}$ is Fredholm if $\epsilon$ is small enough.

If $T_{a_{\epsilon}} f=P\left(a_{\epsilon} f\right)=0$, then $Q\left(a_{\epsilon} f\right)=a_{\epsilon} f-P\left(a_{\epsilon} f\right)=0$ on a set of positive measure and, hence, $Q\left(a_{\epsilon} f\right)=0$. Therefore, $a_{\epsilon} f=0$ and, if $\epsilon$ is small enough (so that $a_{\epsilon} \neq 0$ ) we find that $f=0$ on a set of positive measure. This implies that $f=0$ and we conclude that $T_{a_{\epsilon}}$ is one-to-one. The same is true for the dual operator $\left(T_{a_{\epsilon}}\right)^{*}=T_{\tilde{a}_{\epsilon}}$. Therefore, $T_{a_{\epsilon}}$ is invertible.

Since $T_{a_{\epsilon}}$ is invertible, there is some $f$ so that $T_{a_{\epsilon}} f=P\left(a_{\epsilon} f\right)=1$. Then $Q\left(a_{\epsilon} f\right)=a_{\epsilon} f-P\left(a_{\epsilon} f\right)=-1$ on a set of positive measure and, hence, $Q\left(a_{\epsilon} f\right)=-1$ which is clearly impossible.

Remark 5.5. We do not know whether Fredholmness of $T_{a}$, when $a \in L^{\infty}$ and $Q a \in \mathrm{BMO}_{\log }$, implies invertibility of the symbol in this symbol class, which is optimal in the sense of boundedness.

We next turn our attention to the relation between the symbol class $\mathcal{V}+\overline{H^{\infty}} \cap$ $\mathrm{BMO}_{\log }$ and the space

$$
\mathcal{A}^{1}=\left\{a \in L^{\infty} \cap \mathrm{BMO}_{\log }: H_{a} \in \mathcal{K}\left(H^{1}\right)\right\}
$$

according to the following result.

Theorem 5.6. $\mathcal{A}^{1}=\mathcal{V}+\overline{H^{\infty}} \cap \mathrm{BMO}_{\log }$.

Proof. If $a=v+h$ for some $v \in \mathcal{V}$ and $h \in \overline{H^{\infty}} \cap \mathrm{BMO}_{\log }$, then

$$
H_{a}=H_{v}+H_{h}=H_{v},
$$

which is compact according to Theorem 1.7. On the other hand, if $H_{a}$ is compact, then $P_{1} a \in \mathrm{VMO}_{\log }$ according to Theorem 1.7. Therefore, (7) implies that

$$
a \in \operatorname{lip} \log +\overline{H^{\infty}} \cap \mathrm{BMO}_{\log } \subseteq \mathcal{V}+\overline{H^{\infty}} \cap \mathrm{BMO}_{\log } .
$$

Proposition 5.7. The space $\mathcal{A}^{1}$ is a closed subalgebra of $L^{\infty} \cap \mathrm{BMO}_{\log }$.

Proof. The fact that the space is an algebra follows from Proposition 5.3. Suppose that $a_{n} \rightarrow a$ in $L^{\infty} \cap \mathrm{BMO}_{\log }$ with $a_{n} \in \mathcal{A}^{1}$. Then

$$
\left\|H_{a}-H_{a_{n}}\right\|=\left\|H_{a-a_{n}}\right\| \leq c\left\|P_{1}\left(a-a_{n}\right)\right\|_{\mathrm{BMO}_{\log }} \rightarrow 0
$$

(see Theorem 1.6). Thus, $H_{a}$ is compact.

Corollary 5.8. The space $\mathcal{V}+\overline{H^{\infty}} \cap \mathrm{BMO}_{\log }$ is a Banach algebra.

Proof. This is immediate from the preceding two results. It can also be proved directly. 
The proof of the following theorem is based on an argument of Böttcher and Silberman $[2$, ch. IV] when $1<p<\infty$.

Proposition 5.9. Let $a \in \mathcal{A}^{1}$. Then $T_{a}$ is Fredholm on $H^{1}$ if and only if $a$ is invertible in $\mathcal{A}^{1}$.

Proof. If $a$ is invertible, then formula (27) shows that $T_{a^{-1}}$ is a regularizer of $T_{a}$, and so $T_{a}$ is Fredholm.

If $T_{a}$ is Fredholm, then Theorem 5.4 implies that $a$ is invertible in $L^{\infty} \cap \mathrm{BMO}_{\log }$. Since $T_{a}$ has a regularizer, say $R$, we can write

$$
R T_{a}=I+K,
$$

where $K$ is compact. Therefore, by (28),

$$
0=H_{a a^{-1}}=T_{a} H_{a^{-1}}+H_{a} T_{\tilde{a}^{-1}} .
$$

This implies

$$
H_{a^{-1}}=-K H_{a^{-1}}-R H_{a} T_{\tilde{a}^{-1}}
$$

and, hence, $a^{-1} \in \mathcal{A}^{1}$.

\section{INDEX FORMULA}

For analytic symbols, the Fredholm properties of Toeplitz operators on $H^{1}$ are well understood:

Theorem 6.1. For $a \in \overline{H^{\infty}}$, the Toeplitz operator $T_{a}$ on $H^{1}$ is Fredholm if and only if a is bounded away from zero, in which case

$$
\operatorname{Ind} T_{a}=-\operatorname{ind} a_{r} .
$$

Proof. See [15, Theorem 10].

Our aim in this section is to show that the preceding formula also holds for invertible symbols in the algebra $\mathcal{V}+\overline{H^{\infty}} \cap \mathrm{BMO}_{\log }$. We start with a preliminary lemma.

Lemma 6.2. If $v \in \mathcal{V}$ and $f \in L^{\infty} \cap \mathrm{BMO}_{\log }$, then

$$
\left\|(v f)_{r}-v_{r} f_{r}\right\|_{L^{\infty} \cap \mathrm{BMO}} \longrightarrow 0
$$

as $r \rightarrow 1$.

Proof. From Lemma 2.61 of [3] it follows that $\left\|(v f)_{r}-v_{r} f_{r}\right\|_{\infty} \rightarrow 0$. Therefore, it is enough to show that $\left\|(v f)_{r}-v_{r} f_{r}\right\|_{\mathrm{BMO}_{\log }} \rightarrow 0$. Also, since

$$
\left\|(v f)_{r}-v_{r} f_{r}\right\|_{* *} \leq\left\|(v f)_{r}-v f_{r}\right\|_{* *}+\left\|v f_{r}-v_{r} f_{r}\right\|_{* *},
$$

and $\left\|v-v_{r}\right\|_{\mathrm{BMO}_{\log }} \rightarrow 0$ according to Theorem 2.2, it is sufficient to prove that

$$
\left\|(v f)_{r}-v f_{r}\right\|_{* *} \rightarrow 0
$$

For a function $g: \mathbb{T} \rightarrow \mathbb{C}$, we write

$$
g_{\eta}(\zeta)=g(\zeta \bar{\eta})
$$

when $\zeta, \eta \in \mathbb{T}$. Then

$$
(v f)_{r}(\zeta)-\left(v f_{r}\right)(\zeta)=\frac{1}{2 \pi} \int_{\mathbb{T}} \frac{1-r^{2}}{|1-r \bar{\eta}|^{2}}\left(v_{\eta}(\zeta)-v(\zeta)\right) f_{\eta}(\zeta)|d \eta|
$$


and we need to estimate the expression

$$
\begin{gathered}
J:=\frac{\log \frac{4 \pi}{|I|}}{|I|} \int_{I}\left|(v f)_{r}(\zeta)-\left(v f_{r}\right)(\zeta)-\left((v f)_{r}-v f_{r}\right)_{I}\right||d \zeta| \\
=\frac{\log \frac{4 \pi}{|I|}}{|I|} \int_{I} \mid \frac{1}{2 \pi} \int_{\mathbb{T}} \frac{1-r^{2}}{|1-r \bar{\eta}|^{2}}\left(\left(v_{\eta}(\zeta)-v(\zeta)\right) f_{\eta}(\zeta)\right. \\
\left.\quad-\frac{1}{|I|} \int_{I}\left(v_{\eta}(\theta)-v(\theta)\right) f_{\eta}(\theta)|d \theta|\right) d \eta|| d \zeta \mid \\
\leq \frac{\log \frac{4 \pi}{|I|}}{|I|} \int_{I} \frac{1}{2 \pi} \int_{0}^{2 \pi} \frac{1}{|I|} \int_{I} \frac{1-r^{2}}{|1-r \bar{\eta}|^{2}} \mid\left(v_{\eta}(\zeta)-v(\zeta)\right) f_{\eta}(\zeta) \\
-\left(v_{\eta}(\theta)-v(\theta)\right) f_{\eta}(\theta)|| d \theta|| d \eta|| d \zeta \mid .
\end{gathered}
$$

Write

$$
\begin{aligned}
&\left(v_{\eta}(\zeta)-v(\zeta)\right) f_{\eta}(\zeta)-\left(v_{\eta}(\theta)-v(\theta)\right) f_{\eta}(\theta) \\
&=\left[\left(v_{\eta}(\zeta)-v(\zeta)\right) f_{\eta}(\zeta)-\left(v_{\eta}-v\right)_{I} f_{\eta}(\zeta)\right]+\left[\left(v_{\eta}-v\right)_{I} f_{\eta}(\zeta)-\left(v_{\eta}-v\right)_{I} f_{\eta}(\theta)\right] \\
&+\left[\left(v_{\eta}-v\right)_{I} f_{\eta}(\theta)-\left(v_{\eta}(\theta)-v(\theta)\right) f_{\eta}(\theta)\right]=: J_{1}+J_{2}+J_{3}
\end{aligned}
$$

Then

$$
J \leq \frac{\log \frac{4 \pi}{|I|}}{|I|} \int_{I} \frac{1}{2 \pi} \int_{0}^{2 \pi} \frac{1}{|I|} \int_{I} \frac{1-r^{2}}{|1-r \bar{\eta}|^{2}}\left(\left|J_{1}\right|+\left|J_{2}\right|+\left|J_{3}\right|\right)|d \theta||d \eta||d \zeta| .
$$

Let us first consider $J_{1}$. We have

$$
\begin{aligned}
& \frac{\log \frac{4 \pi}{|I|}}{|I|} \int_{I} \frac{1}{2 \pi} \int_{0}^{2 \pi} \frac{1}{|I|} \int_{I} \frac{1-r^{2}}{|1-r \bar{\eta}|^{2}}\left|J_{1}\right||d \theta||d \eta||d \zeta| \\
& \leq \frac{\|f\|_{\infty}}{2 \pi} \int_{0}^{2 \pi} \frac{1-r^{2}}{|1-r \bar{\eta}|^{2}} \frac{\log \frac{4 \pi}{|I|}}{|I|} \int_{I}\left|v_{\eta}(\zeta)-v(\zeta)-\left(v_{\eta}-v\right)_{I}\right||d \zeta||d \eta| \\
& \leq \frac{\|f\|_{\infty}}{2 \pi} \int_{0}^{2 \pi} \frac{1-r^{2}}{|1-r \bar{\eta}|^{2}}\left\|v_{\eta}-v\right\|_{* *}|d \eta| \\
& =\frac{\|f\|_{\infty}}{2 \pi} \int_{0<|1-\eta|<\delta} \frac{1-r^{2}}{|1-r \bar{\eta}|^{2}}\left\|v_{\eta}-v\right\|_{* *}|d \eta| \\
& +\frac{\|f\|_{\infty}}{2 \pi} \int_{\delta<|1-\eta|<2} \frac{1-r^{2}}{|1-r \bar{\eta}|^{2}}\left\|v_{\eta}-v\right\|_{* *}|d \eta| .
\end{aligned}
$$

Now given $\epsilon>0$, there is $\delta>0$ (according to Theorem 2.2) and $r<1$ such that the above sum of two integrals can be estimated above by

$$
\frac{\|f\|_{\infty}}{2 \pi} 2 \pi \epsilon+\frac{\|f\|_{\infty}}{2 \pi} \epsilon 2\|v\|_{* *} .
$$

Similarly the part made of $J_{3}$ can be shown to be as small as we wish provided that $r$ is sufficiently close to 1 .

It remains to consider $J_{2}$. Note that

$$
\begin{aligned}
& \left(v_{\eta}-v\right)_{I} f_{\eta}(\zeta)-\left(v_{\eta}-v\right)_{I} f_{\eta}(\theta) \\
& =\left(v_{\eta}-v\right)_{I}\left(f_{\eta}(\zeta)-\left(f_{\eta}\right)_{I}\right)+\left(v_{\eta}-v\right)_{I}\left(\left(f_{\eta}\right)_{I}-f_{\eta}(\theta)\right)
\end{aligned}
$$


and that it is sufficient to consider only one of the terms in the equality above. Since, by the choice of $\delta>0$ and $r<1$,

$$
\begin{aligned}
& \frac{1}{2 \pi} \int_{0}^{2 \pi} \frac{1-r^{2}}{|1-r \bar{\eta}|^{2}}\left|v_{\eta}-v\right|_{I} \frac{\log \frac{4 \pi}{|I|}}{|I|} \int_{I}\left|f_{\eta}(\zeta)-\left(f_{\eta}\right)_{I}\right||d \zeta||d \eta| \\
& \leq \frac{\|f\|_{* *}}{2 \pi}\left(\int_{0<|1-\eta|<\delta} \frac{1-r^{2}}{|1-r \bar{\eta}|^{2}}\left|v_{\eta}-v\right|_{I}|d \eta|\right. \\
& +\int_{\delta<|1-\eta|<2} \frac{1-r^{2}}{|1-r \bar{\eta}|^{2}}\left|v_{\eta}-v\right|_{I}|d \eta|
\end{aligned}
$$

can be made arbitrarily small, the proof is complete.

Theorem 6.3. If $a, b \in \mathcal{V}+\overline{H^{\infty}} \cap \mathrm{BMO}_{\log }$, then

$$
\left\|(a b)_{r}-a_{r} b_{r}\right\|_{L^{\infty} \cap \mathrm{BMO}_{\log }} \longrightarrow 0 \text { as } r \rightarrow 1
$$

Proof. The statement follows easily from the preceding lemma and the fact $(h g)_{r}=$ $h_{r} g_{r}$ for $h, g \in \overline{H^{\infty}}$.

Proof of the index formula. According to Lemma 5.1 and the general theory of Fredholm operators, there is a function $\chi_{n} h\left(n \geq 0\right.$ and $\left.h \in \overline{H^{\infty}}\right)$ that has the same index as $a$ and generates a Toeplitz operator that is Fredholm of the same index as $T_{a}$. Using Theorems 6.1 and 6.3, and well-known properties of the index (of Fredholm operators and of continuous functions), and [14, Lemma 5] saying that Ind $T_{\chi_{n}}=-n$, we can conclude that for $r$ sufficiently close to 1 , we have

$$
\begin{aligned}
\text { Ind } T_{\chi_{n} h} & =\operatorname{Ind} T_{\chi_{n}}+\operatorname{Ind} T_{h} \quad \text { (Atkinson) } \\
& =-n-\operatorname{ind} h_{r} \\
& =-\operatorname{ind}\left(\left(\chi_{n}\right)_{r} h_{r}\right) \\
& =-\operatorname{ind}\left(\chi_{n} h\right)_{r} \\
& =-\operatorname{ind} a_{r} .
\end{aligned}
$$

Proof of Theorem 1.8. Indeed this is an immediate consequence of Propositions 5.2 and 5.9, Theorem 5.6, and the preceding proof of the index formula.

\section{REFERENCES}

[1] A. Böttcher and Y. Karlovich, Carleson curves, Muckenhoupt weights, and Toeplitz operators. Progress in Mathematics 154, Birkhäuser Verlag, Basel, 1997.

[2] A. Böttcher and B. Silbermann, Invertibility and asymptotics of Toeplitz matrices. AkademieVerlag, Berlin, 1983.

[3] A. Böttcher and B. Silbermann, Analysis of Toeplitz operators. 2nd edition, Springer-Verlag, Berlin and Heidelberg, 2006.

[4] J. Cima and D. Stegenga, Hankel operators on $H^{p}$. Analysis at Urbana. Vol. 1: Analysis in function spaces, London Mathematical Society Lecture Note Series, 137, Cambridge University Press, Cambridge (1989), 133-150.

[5] L. A. Coburn, Weyl's Theorem for non-normal operators. Michigan Math. J. 13 (1966), 285-286.

[6] R. G. Douglas, Toeplitz and Wiener-Hopf operators in $H^{\infty}+C$. Bull. Amer. Math. Soc. 74 (1968), 895-899.

[7] R. G. Douglas, Banach algebra techniques in operator theory. 2nd edition, Springer-Verlag, New York, 1998.

[8] J. B. Garnett, Bounded Analytic Functions. Revised 1st edition, Springer-Verlag, 2007. 
[9] S. Janson, On functions with conditions on the mean oscillation. Ark. Mat. 14, N2 (1976), 189-196.

[10] S. Janson, J. Peetre and S. Semmes, On the action of Hankel and Toeplitz operators on some function spaces. Duke Math. J. 51, no. 4 (1984), 937-958.

[11] I. Katznelson, An introduction to harmonic analysis. 3rd edition, Cambridge University Press, New York, 2004.

[12] D. A. Stegenga, Bounded Toeplitz operators on $H^{1}$ and applications of the duality between $H^{1}$ and the functions of bounded mean oscillation. Amer. J. of Math. 98, no. 3 (1976), 573-589.

[13] V. A. Tolokonnikov, Hankel and Toeplitz operators in Hardy spaces (Russian. English summary). Investigations on linear operators and the theory of functions, XIV, Zap. Nauchn. Sem. Leningrad. Otdel. Mat. Inst. Steklov. (LOMI) 141 (1985), 165-175. (English translation in J. Sov. Math. 37 (1987), 1359-1364.)

[14] J. A. Virtanen, Fredholm theory of Toeplitz operators on the Hardy space $H^{1}$. Bull. London Math. Soc. 38 (2006), 143-155.

[15] D. Vukotić, Analytic Toeplitz operators on the Hardy space $H^{p}$ : a survey. Bull. Belg. Math. Soc. 10 (2003), 101-113.

Department of Mathematics, University of Crete, Knossos Avenue, 71409 Iraklion, GreECE

E-mail address: papadim@math.uoc.gr

Department of Mathematics, University of Crete, Knossos Avenue, 71409 Iraklion, Greece, Current address:, Department of Mathematics, University of Helsinki, Gustaf Hällströmin katu 2 B, 00014 Helsinki, Finland

E-mail address: jani.virtanen@helsinki.fi 